\title{
A COMPARISON OF TIME DOMAIN BOUNDARY CONDITIONS FOR ACOUSTIC WAVES IN WAVE GUIDES
}

\author{
$\mathrm{BY}$ \\ H. T. BANKS (Center for Research in Scientific Computation, North Carolina State University, Raleigh), \\ G. PROPST (Institut für Mathematik, Universität Graz, Austria), \\ AND
}

R. J. SILCOX (Acoustics Division, NASA Langley Research Center, Hampton, VA)

\begin{abstract}
We consider several types of boundary conditions in the context of time domain models for acoustic waves. Experiments with four different duct terminations (hardwall, free radiation, foam, wedge) were carried out in a wave duct from which reflection coefficients over a wide frequency range were measured. These reflection coefficients are used to estimate parameters in the time domain boundary conditions, and a comparison of the relative merits of the models in describing the data is presented. Boundary conditions that yield a good fit of the model to the experimental data were found for all duct terminations except the wedge.
\end{abstract}

1. Introduction. Traditional techniques for modeling the sound fields in ducts and enclosures utilize an impedance concept. This approach is quite useful for harmonic sound fields as well as quantification of steady state, random sound fields in the frequency domain. However, in solving for a transient response, impedance concepts which are defined only for steady state conditions in the frequency domain are not applicable. Impedance-based models provide a way to easily quantify the acoustic properties of the boundary as a function of the various parameters characterizing the acoustic interface. Generally an acoustic impedance boundary condition is found to be a function of frequency and the spatial position along the boundary. In addition, the boundary condition is based on assumptions of linearity as well as the assumption that the boundary surface is locally reacting [1, pp. 257-270]. The first assumption is questionable at sufficiently high sound pressure levels and the latter applies for resonator designs but not for the more generally used bulk reacting materials such as foams and fiberglass.

The need for boundary conditions formulated in the time domain is a well publicized need [2] that has been unresolved for years. Generally, only total reflection or absorption

Received July 20, 1993.

1991 Mathematics Subject Classification. Primary 35A05, 35L05, 35R30, 35Q99.

Research supported in part (H.T.B.) by the Air Force Office of Scientific Research under grant AFOSR90-0091. This research was carried out in part while the first two authors were visiting scientists at the Institute for Computer Applications in Science and Engineering (ICASE), NASA Langley Research Center, Hampton, VA 23665, which is operated under NASA Contract No. NAS1-18107. 
(being constant with frequency) have been formulated as boundary conditions in the time domain [3]. This includes the applications of Sommerfield's radiation condition in the free field. One approach to avoid the imposition of termination conditions in ducts is to couple the radiation problem to the duct problem [4]. This however comes at great computational complexity and cost and again has only been applied to a harmonic analysis. Other approaches have resorted to filtering the reflections [5] or applying Cepstral analysis to remove unwanted reflections from an analysis after the initial calculation [6]. Bolton [7] utilized a time domain technique to define the normal impedance of foam materials. The total pressure above the impedance surface exposed to a normally incident plane wave was measured using a specialized time waveform. Using Cepstral techniques, the impulse response of the reflective surface was deconvolved from the excitation time history. This effectively confirms the compatibility of the boundary conditions in the time and frequency domains.

The efforts reported here are the first steps in the development of state space/time domain models for use in control design problems related to active control of noise in a closed cylinder. The ultimate intent is to model the frequency-dependent impedance of a treated aircraft interior such that the time domain interior pressure response to transient excitation may be predicted. In such applications, one has negligible fluid damping of the acoustic pressure fields. Since the major dissipative mechanism entails the partial absorption/partial reflection that occurs at the fluid/wall interface, it is important in the control of the interior acoustic pressure to model this dissipation accurately.

One objective of our contribution here is achieved in Sec. 2. This entails relating specific boundary conditions in time domain/state space models with corresponding reflection coefficients arising in the case of simple harmonic waves in a frequency-domain approach. Moreover, we summarize the absorptive/reflective characteristics of the boundary conditions (and corresponding reflection coefficients) as a function of the parameters in the boundary conditions. The boundary condition models can be formulated to treat general three-dimensional acoustic pressure fields. However, in a first comparison with data, we restrict our experimental evaluation to the one-dimensional plane waves. This gives some indication of the validity of the various boundary models when applied to different terminations. It, of course, does not validate the general boundary conditions for the multi-dimensional situation.

A logical approach to modeling locally reacting boundaries is the use of a mass-springdamper model [8], [9] coupled at the boundary to the acoustic space. This accounts for the time delay and resistance (damping) inherent in real acoustic boundaries. Extensions to more complicated systems may be made by making the mechanical system parameters a function of position. Additionally, multiple resonances may be represented as multiple coupled mechanical systems. Similarly, nonlinear effects may be modeled using nonlinear mechanical elements (spring and damper model). This paper presents an initial investigation into this approach by modeling frequency-dependent impedance boundary conditions with a number of different simple mechanical systems. Results are presented comparing the measured values of impedance to the values modeled, after a least means squares fit of the model parameters to the data is performed. Validation results are restricted to plane waves for this initial evaluation. However, rather than using predicted 
impedances, measured results are provided that are typical of real-world materials and conditions. These provide a more rigorous evaluation of the modeling capability of these simple mechanical systems.

2. Boundary conditions and reflection coefficients. The physical quantities used for the description of acoustic wave motion in a fluid are the acoustic pressure $p$ (being the deviation from the mean pressure at equilibrium) and the velocity potential $\phi$. For waves of small amplitude, to first order both $p$ and $\phi$ satisfy the linear undamped wave equation with uniform speed of sound $c$ in the fluid, where $c^{2}=1 /(\rho K), \rho$ being the equilibrium density of the fluid and $K$ being the adiabatic compressibility of the fluid at equilibrium. The velocity potential $\phi$ is a complex-valued function such that $v(t, x)=-\nabla \phi(t, x)$ is the fluid's velocity at $x$ at any time $t$. The acoustic pressure is related to the velocity potential by $p(t, x)=\rho \phi_{t}(t, x)[1, \mathrm{pp} .243-257]$.

We consider three types of boundary conditions for the wave equation and derive the corresponding reflection coefficients for simple harmonic waves in a one-dimensional wave guide by insertion of the superposition of right and left propagating waves into the boundary conditions. The reflection coefficients are of the form $R=(z-\rho c) /(z+\rho c)$ where $z$ is the acoustic impedance of the boundary surface [1, pp. $259 \mathrm{ff}$.].

2.1. Oscillating boundaries. In [8], Beale considers a bounded region $\Omega \subset \mathbf{R}^{3}$ where the interaction of the fluid with the boundary material at the (sufficiently smooth) boundary $\partial \Omega$ is modeled by a continuum of damped harmonic oscillators. Within $\Omega$, the velocity potential $\phi(t, x)$ satisfies the wave equation

$$
\phi_{t t}(t, x)=c^{2} \nabla^{2} \phi(t, x), \quad x \in \Omega
$$

For every $x \in \partial \Omega$, the normal displacement $\delta(t, x)$ of the boundary into the fixed domain $\Omega$ is assumed to be independent of other parts of the boundary surface (a surface of local reaction, $[1$, p. 260]). With the effective mass $m(x)$, the resistance $d(x)$, and the stiffness $k(x)$ per unit area of the boundary surface, the interaction of the boundary and the interior pressure is described by

$$
m(x) \delta_{l t}(t, x)+d(x) \delta_{t}(t, x)+k(x) \delta(t, x)=-\rho \phi_{t}(t, x), \quad x \in \partial \Omega
$$

In addition, from the assumption that the surface is impenetrable by the fluid it follows that the velocity of the fluid and of the boundary coincide, i.e.,

$$
\delta_{t}(t, x)=\phi_{n}(t, x), \quad x \in \partial \Omega
$$

where $\phi_{n}=\partial \phi / \partial n$ denotes the outward (to the domain $\Omega$ ) normal derivative of $\phi$.

The system (2.1)-(2.3) can be formulated as an abstract Cauchy problem for the state $u=\left(\phi, \phi_{t}, \delta, \delta_{t}\right)$ in the space $H=\bar{H}^{1}(\Omega) \times L^{2}(\Omega) \times L^{2}(\partial \Omega) \times L^{2}(\partial \Omega)$. Here, as usual, $L^{2}(M)$ denotes the Lebesgue space of square integrable functions on $M \subseteq \mathbf{R}^{3}$ and $\bar{H}^{1}(\Omega)$ is the quotient of the Sobolev space $H^{1}(\Omega)$ over the set of constant functions $\left(H^{m}(\Omega)\right.$ is the space of functions having distributional derivatives up to order $m$ in $L^{2}(\Omega)$ ). With 
the norm $|\cdot|_{H}$ representing the energy of system (2.1)-(2.3) at any fixed time $t$, which is given by

$$
|u|_{H}^{2}=\int_{\Omega}\left(\rho|\nabla \phi|^{2}+\rho c^{-2}\left|\phi_{t}\right|^{2}\right) d x+\int_{\partial \Omega}\left(k|\delta|^{2}+m\left|\delta_{t}\right|^{2}\right) d S
$$

$H$ is a Hilbert space. For $u(t) \in D(A)$, where

$$
D(A) \equiv\left\{\left(u_{1}, u_{2}, u_{3}, u_{4}\right) \in H \mid \nabla^{2} u_{1} \in L^{2}(\Omega), u_{2} \in H^{1}(\Omega), u_{1 n}=u_{4} \text { on } \partial \Omega\right\},
$$

the system (2.1)-(2.3) can be written in the form $\frac{d}{d t} u(t)=A u(t)$ with the linear operator $A$ defined by

$$
A\left(u_{1}, u_{2}, u_{3}, u_{4}\right)=\left(u_{2}, c^{2} \nabla^{2} u_{1}, u_{4},-\left(\rho u_{2}+k u_{3}+d u_{4}\right) / m\right) .
$$

In [8] the well-posedness of system $(2.1) \cdots(2.3)$ is established by proving that $A$ with domain $D(A)$ is the generator of a $C_{0}$ contraction semigroup on $H$, provided that $m(x)>$ $0, k(x)>0$, and $d(x) \geq 0$ for each $x \in \partial \Omega$. An analysis of the spectrum is given and, in particular, Beale's considerations reveal that one cannot obtain an exponential decay bound for the semigroup even in the case where $d>0$.

We consider the one-dimensional case $\Omega=(-\ell, 0)$ where the boundary at 0 is characterized by three oscillator constants $m, d, k \in \mathbf{R}$. The general solution to the wave equation in $(-\ell, 0)$ is the superposition of right and left propagating waves. If $\phi(t, x)=$ $F(t-x / c)+G(t+x / c)$ and $\delta(t)$ are (sufficiently smooth) solutions to the system (2.1)$(2.3)$ on $(-\ell, 0)$, they satisfy the condition

$$
\begin{gathered}
m \delta_{t t}(t)+d \delta_{t}(t)+k \delta(t)=-\rho \phi_{t}(t, 0), \\
\delta_{t}(t)=\phi_{x}(t, 0) .
\end{gathered}
$$

Computing $\delta$ and $\delta_{t t}$ by integration (resp. differentiation) of (2.5) we obtain

$$
\begin{gathered}
\delta(t)=-\frac{1}{c} \int^{t}\left(F^{\prime}(\tau)-G^{\prime}(\tau)\right) d \tau=-(F(t)-G(t)) / c+\text { const. } \\
\delta_{t t}(t)=-\left(F^{\prime \prime}(t)-G^{\prime \prime}(t)\right) / c
\end{gathered}
$$

where the constant is set to zero because for $F(t) \equiv G(t) \equiv 0$ the boundary should be at equilibrium $\delta(t) \equiv 0$. Inserting this into (2.4), we find

$$
m G^{\prime \prime}(t)+(d+\rho c) G^{\prime}(t)+k G(t)=m F^{\prime \prime}(t)+(d-\rho c) F^{\prime}(t)+k F(t)
$$

for the coupling of $F$ and $G$ by the oscillator at $x=0$.

Suppose that $F$, the wave incident on the boundary, is a simple harmonic of frequency $\omega /(2 \pi)$, i.e., $F(t-x / c)=\exp \{i \omega(t-x / c)\}$. Then the right side of $(2.6)$ is a harmonic forcing function for the linear oscillator (2.6). It follows (neglecting possible transients due to initial conditions that we do not specify here; see, e.g., [1, p. 45]) that the steady state of $G$ is harmonic with the same frequency $\omega /(2 \pi)$, i.e., $G(t+x / c)=$ 
$R(\omega) \exp \{i \omega(t+x / c)\}$ with some complex constant $R(\omega)$. Inserting $F, G$, and their derivatives into $(2.6)$ we then obtain

$$
R(\omega)=\frac{m \omega^{2}-i \omega(d-\rho c)-k}{m \omega^{2}-i \omega(d+\rho c)-k} .
$$

This formula for the reflection coefficient coincides with (6.3.8) of [1], where it is derived from equating the acoustic impedance $p / v_{\text {incident }}$ with the impedance $z(\omega)=i \omega m+d+$ $k /(i \omega)$ of the oscillating boundary, which implies $R(\omega)=(z(\omega)-\rho c) /(z(\omega)+\rho c)$.

To sum up, in the one-dimensional version of the model (2.1)-(2.3), a simple harmonic wave is reflected at the boundary by reversion of its direction of propagation, multiplication of its amplitude with $|R(\omega)|$ and a shift of its phase by $\arg R(\omega)$. The magnitude of $R(\omega)$ is smaller than one if and only if $d>0$. In this case the oscillating boundary absorbs acoustic energy. In the case $d=0$, energy is conserved. In state space (time domain) terminology, we have (see [8]) that for $d>0, A$ is dissipative and the infinitesimal generator of a $C_{0}$ contraction semigroup (i.e., $S(t)=e^{A t}$ where $|S(t)|_{H} \leq 1$ ) so that acoustic energy can be absorbed at the boundary while if $d=0, S(t)$ is a unitary group and no energy is absorbed at the boundary (energy is conserved). However, even in the case that $d>0$, one does not have an exponential decay bound for solutions (i.e., there does not exist a $\lambda>0$ such that $|S(t)|_{H} \leq M e^{-\lambda t}$ ).

2.2. Damped elastic boundaries. For $d=0, k=0$ the model $(2.2),(2.3)$ together with $p=\rho \phi_{t}$ results in the boundary condition

$$
m(x) p_{n}(t, x)+\rho p(t, x)=0, \quad x \in \partial \Omega
$$

for the acoustic pressure $p$. This is called a Robin or elastic boundary condition. To include dissipation it is extended by adding a $p_{t}$ term, which gives systems that are studied, for example, in [10], [11], [12]. In [12] the wave equation for the acoustic pressure

$$
p_{t t}(t, x)=c^{2} \nabla^{2} p(t, x), \quad x \in \Omega
$$

with damped elastic boundary conditions

$$
\alpha p(t, x)+\beta p_{t}(t, x)+c p_{n}(t, x)=0, \quad x \in \partial \Omega
$$

is the model that is used for investigating active noise control techniques.

For $\alpha>0, \beta \geq 0$ the linear operator $A$ defined by

$$
A(f, g)=\left(g, c^{2} \nabla^{2} f\right)
$$

with domain

$$
D(A)=\left\{(f, g) \in H^{2}(\Omega) \times H^{1}(\Omega) \mid \alpha f+\beta g+c f_{n}=0 \text { on } \partial \Omega\right\}
$$

is the generator of a $C_{0}$ contraction semigroup corresponding to problem (2.7)-(2.8) with the state $\left(p, p_{t}\right)$ in the space $H^{1}(\Omega) \times L^{2}(\Omega)$ taken with the $H^{1}(\Omega) \times L^{2}(\Omega)$-equivalent norm $|\cdot|_{\mathcal{H}}$ corresponding to the inner product

$$
\left\langle\left(f_{1}, g_{1}\right),\left(f_{2}, g_{2}\right)\right\rangle_{\mathcal{H}}=\frac{\alpha}{c} \int_{\partial \Omega} f_{1} f_{2}+\int_{\Omega \Omega} \nabla f_{1} \nabla f_{2}+\frac{1}{c^{2}} \int_{\Omega} g_{1} g_{2}
$$


(see [12]). Note that the semigroup is not a contraction in the usual $H^{1}(\Omega) \times L^{2}(\Omega)$ norm.

To obtain reflection coefficients for harmonic waves in the one-dimensional case $\Omega=$ $(-\ell, 0)$ we insert the superposition $p(t, x)=F(t-x / c)+G(t+x / c)$ into the condition $\alpha p(t, 0)+\beta p_{t}(t, 0)+c p_{x}(t, 0)=0$, and this yields

$$
(1+\beta) G^{\prime}(t)+\alpha G(t)=(1-\beta) F^{\prime}(t)-\alpha F(t) .
$$

Thus, if the incident wave $F(t-x / c)=\exp \{i \omega(t-x / c)\}$ is a simple harmonic, so is the reflected wave $G(t+x / c)=R(\omega) \exp \{i \omega(t+x / c)\}$ (aside from transients) with the reflection coefficient

$$
R(\omega)=\frac{i \omega(1-\beta)-\alpha}{i \omega(1+\beta)+\alpha}
$$

This equals $(z(\omega)-\rho c) /(z(\omega)+\rho c)$ with $z(\omega)=i \omega \rho c /(\alpha+i \omega \beta)$.

This shows that (ELAST) models a boundary surface with specific acoustic impedance $[1$, p. 261] given by $\zeta \equiv z /(\rho c)=i \omega /(\alpha+i \omega \beta)$. The magnitude of the reflection is smaller than 1 if and only if $\beta>0$. In the case $\beta=0$ the impedance is purely imaginary and the amplitude of the reflected wave is equal to the amplitude of the incident one. In the state-space or time-domain formulation we have that for $\beta \geq 0$ the reflecting surface produces a contraction semigroup if the norm $|\cdot|_{\mathcal{H}}$ for $H^{1}(\Omega) \times L^{2}(\Omega)$ as indicated above is chosen. In actual fact, if $\beta>0$ the damping is sufficiently strong so that one has an exponential bound in the $\mathcal{H}$-induced operator norm: $|S(t)|_{\mathcal{H}} \leq M_{0} e^{-\mu_{0} t}$ for $M_{0}, \mu_{0}>0$; see $[12]$.

2.3. Frequency-independent boundaries. For $m \equiv 0$ and $k \equiv 0$, the model $(2.2),(2.3)$ reduces to

$$
-d(x) \phi_{n}(t, x)=\rho \phi_{t}(t, x), \quad x \in \partial \Omega
$$

This equation states that the acoustic impedance $p / v_{\text {incident }}$ at the boundary equals $d(x)$. Equation (2.1) with boundary conditions of the form (2.9) is the subject of many publications throughout the literature on the wave equation. For aspects concerning well-posedness, decay of solutions or control problems see, for example, [13], [14], [15]. The report [16] summarizes some results on the one-dimensional case $\Omega=(-\ell, 0)$ of $(2.1)$ with boundary conditions (which we refer to as impedance boundary conditions) of the form

$$
\phi_{t}(t, 0)+\zeta c \phi_{x}(t, 0)=0
$$

with complex specific acoustic impedance $\zeta$ of the boundary surface. In case $\operatorname{Re} \zeta \geq 0$ the operator

$$
A(w, p)=c\left(p, w^{\prime \prime}\right)
$$

with domain

$$
D(A)=\left\{(w, p) \mid(w, p) \in H^{2}(-\ell, 0) \times H^{1}(-\ell, 0), p+\zeta w_{n}=0 \text { at }-\ell \text { and } 0\right\}
$$

generates a $C_{0}$ contraction semigroup $S(t)$ corresponding to the state $\left(c \phi, \phi_{t}\right)$ in a space $H_{E}$. The Hilbert space $H_{E}$ is the product of the quotient space $\bar{H}^{1}(-\ell, 0)$ (i.e., the 
quotient of $H^{1}$ over the set of constant functions) with $L^{2}(-\ell, 0)$. The norm $|(w, p)|_{H_{E}}^{2}=$ $\left|w^{\prime}\right|_{L^{2}}^{2}+|p|_{L^{2}}^{2}$ in $H_{E}$ corresponds to the acoustic energy of the state.

Substituting $\phi(t, x)=F(t-x / c)+G(t+x / c)$ into $(2.10)$, we obtain $G(t)=$ $\{(\zeta-1) /(\zeta+1)\} F(t)+$ const. (The constant here is set to 0 , because $F \equiv 0$ should imply $G \equiv 0$ (passive boundary)). Thus, the reflection coefficient for this model

$$
R(w)=R=\frac{\zeta-1}{\zeta+1}
$$

does not depend on the shape of the reflected waves. Moreover, $\operatorname{Re} \zeta \geq 0$ is equivalent to $|R| \leq 1$, with $|R|<1$ if and only if $\operatorname{Re} \zeta>0$. Thus, no acoustic energy is absorbed at the boundary if $\zeta$ is purely imaginary. If $\zeta=1$, then $R=0$ and we have a totally absorbing boundary. In the state space formulation (see [16]) we find that for $\operatorname{Re} \zeta \geq 0$, $A$ is dissipative and generates a contraction semigroup $S(t)$. Indeed, for $\zeta \neq \pm 1$, we can argue that $A$ is in fact a spectral operator and $S(t)$ can be expanded in a Riesz basis of eigenfunctions. For $\operatorname{Re} \zeta>0$, energy is absorbed at the boundary and we obtain an exponential bound for $S(t)$. For $\zeta=1$, the spectrum of $A$ is empty and, moreover, we can argue that we have a totally absorbing boundary. Thus, we find that the state-space formulation and the usual frequency domain considerations lead to similar characterizations in terms of the parameter $\zeta$.

2.4. Remarks. As we have seen in the discussions above, the three types of boundary conditions can readily be related to one another. However, we should note that none of these boundary conditions can be viewed as a special case of the others. While the oscillating boundary conditions (OSCIL) of Sec. 2.1 may appear to be the most general case, they do not contain either the damped elastic conditions (ELAST) of Sec. 2.2 or the frequency-independent conditions (IMPED) of Sec. 2.3 as special cases. In the event we consider (ELAST) with no damping (i.e., $\beta=0$ ), then we do obtain a special case of (OSCIL) by taking $d=0, k=0$, and $\alpha=\rho c / m$ as can be easily seen by consideration of the boundary conditions (2.8) and (2.2) directly or the reflection coefficients in (OSCIL) and (ELAST). The impedance boundary conditions of Sec. 2.3--see (2.10)-are a special case of (OSCIL) with $m=0, k=0$ only if we restrict the specific acoustic impedance $\zeta$ of the boundary surface to be a real number. Neither of these special conditions on the parameters of (ELAST) or (IMPED) is typical of the standard use of these boundary conditions in the acoustics literature. That these boundary conditions are indeed each quite distinct is manifested in the numerical findings for the fits to experimental data of Sec. 4, where it is seen that no one of the boundary conditions is superior to the other two for all types of terminations.

\section{Experiments and data analysis.}

3.1. Experimental procedures. The procedure used to determine the reflection coefficient of the various duct termination conditions is similar to that outlined in reference [17]. The model for planar wave propagation in a duct represents the pressure anywhere in the duct as defined by the following equation:

$$
p(t, x)=A(\omega)\{\exp [i(\omega t-k x)]+R(\omega) \exp [i(\omega t+k x)]\} .
$$




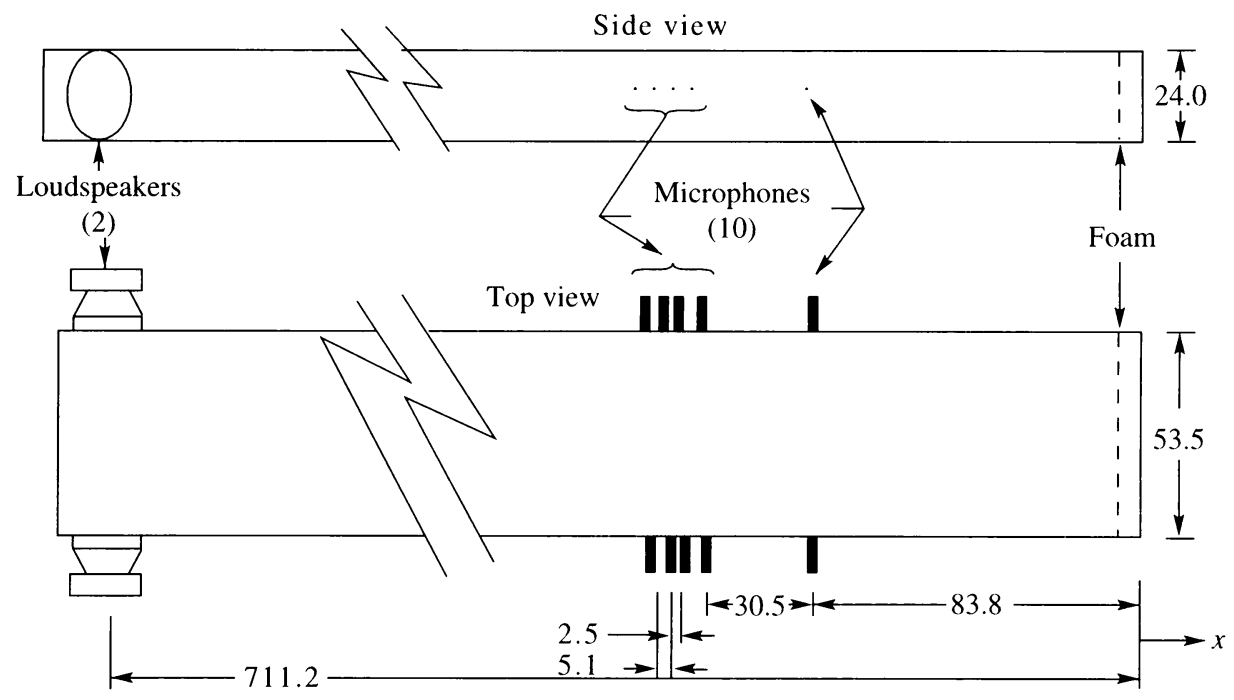

FIG. 1

In this expression, $t$ and $x$ represent the temporal and spatial variables, respectively, $p$ is the measured acoustic pressure relative to a common phase reference, $A(\omega)$ is the unknown incident wave amplitude and $R(\omega)$ is the unknown complex reflection coefficient. The wave number, $\omega / c$, is represented by $k$, and $\omega$ is the angular frequency.

By measuring the pressure, $p\left(t, x_{j}\right)$, at a number of axial locations, $x_{j}$, an overdetermined set of equations may be formed using Eq. (3.1) and solved in a least squares sense. The variables solved for in this analysis are the positive and negative complex wave amplitudes represented by $A(\omega)$ and $A(\omega) R(\omega)$. A schematic of the test configuration used in three of the test cases is shown in Fig. 1. Ten microphone locations were utilized at five different axial locations. By making measurements in pairs on directly opposite sides of the duct and adding the two complex measurements, the contribution due to the first higher-order mode is summed out. The resulting measurements at the five axial locations noted in the figure are used in conjunction with Eq. (3.1).

The data was acquired in the frequency domain using pseudo-random excitation of the acoustic sources shown in the left part of Fig. 1. This harmonic excitation allowed the wave field to be defined with two hertz resolution over a bandwidth to $650 \mathrm{hz}$. By exciting the duct with symmetrical excitation on opposite sides of the duct, the dominant wave generated was planar even above the cut-on frequency of the first cross mode at $325 \mathrm{hz}$. This in combination with the microphone averaging technique outlined above allowed the frequency range to extend to the cut-on frequency of the second higher-order mode at $650 \mathrm{hz}$.

For this configuration, three termination conditions were investigated. The first is a near hardwall condition attained by terminating the duct with a 0.5 inch thick reinforced aluminum plate. For a true hardwall termination. the reflection coefficient would be expected to be invariant with frequency and to be purely real with a value of 1.0 . This 


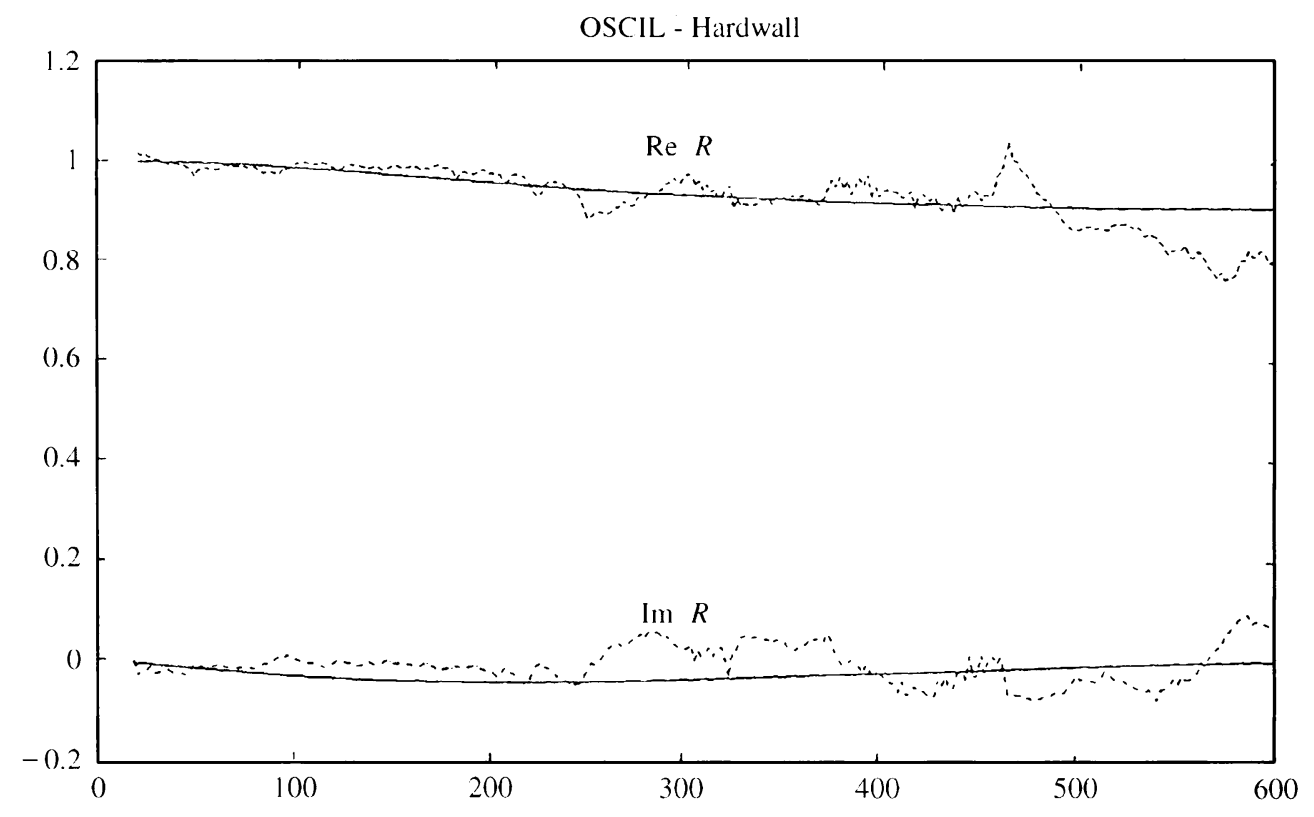

Frequency in hz

FIG. $2 a$

panel has a fundamental resonance at about $250 \mathrm{hz}$ and another at $450 \mathrm{hz}$ and therefore appears somewhat soft at these frequencies where minor variations from the hardwall condition are observed in the data. The data in Fig. 2 (in all figures, the data will be given by a dashed line) reveals a reflection coefficient with real part varying from 1.0 at $20 \mathrm{hz}$ to about 0.8 at $600 \mathrm{hz}$, while the imaginary part is near zero with variations between -0.1 and 0.1 in the range $20 \mathrm{hz}$ to $600 \mathrm{hz}$.

The second case was for free radiation from an open duct. This case displays a frequency dependence as a plane wave at low frequency would reflect from a pressure release boundary with a purely real reflection coefficient of -1.0 . However, at the high frequency limit, all of the propagating energy wave would beam out of the end of the open duct and the reflection coefficient should decrease to zero. The real part of the reflection coefficient data shown in Fig. 3 (see p. 259) exhibits this general trend. However, the imaginary part of the measured data varies over a positive range from 0.2 to 0.6 . This behavior may be attributed to the presence of several reflecting surfaces outside of the duct but in the general vicinity of the duct exit. Also, the room was generally reverberant and may have exhibited some modal response.

The final case tested in the duct of Fig. 1 was an egg-crate foam referred to as a wedge. This foam was $5.08 \mathrm{~cm}$ thick from tip to base and was backed by an additional $10.16 \mathrm{~cm}$ of closed-cell foam. The back side of this foam was left open to the laboratory space. It is hard to anticipate the exact behavior of this type of termination condition. However, it is expected to have a generally complex reflection with a reflection coefficient that is 


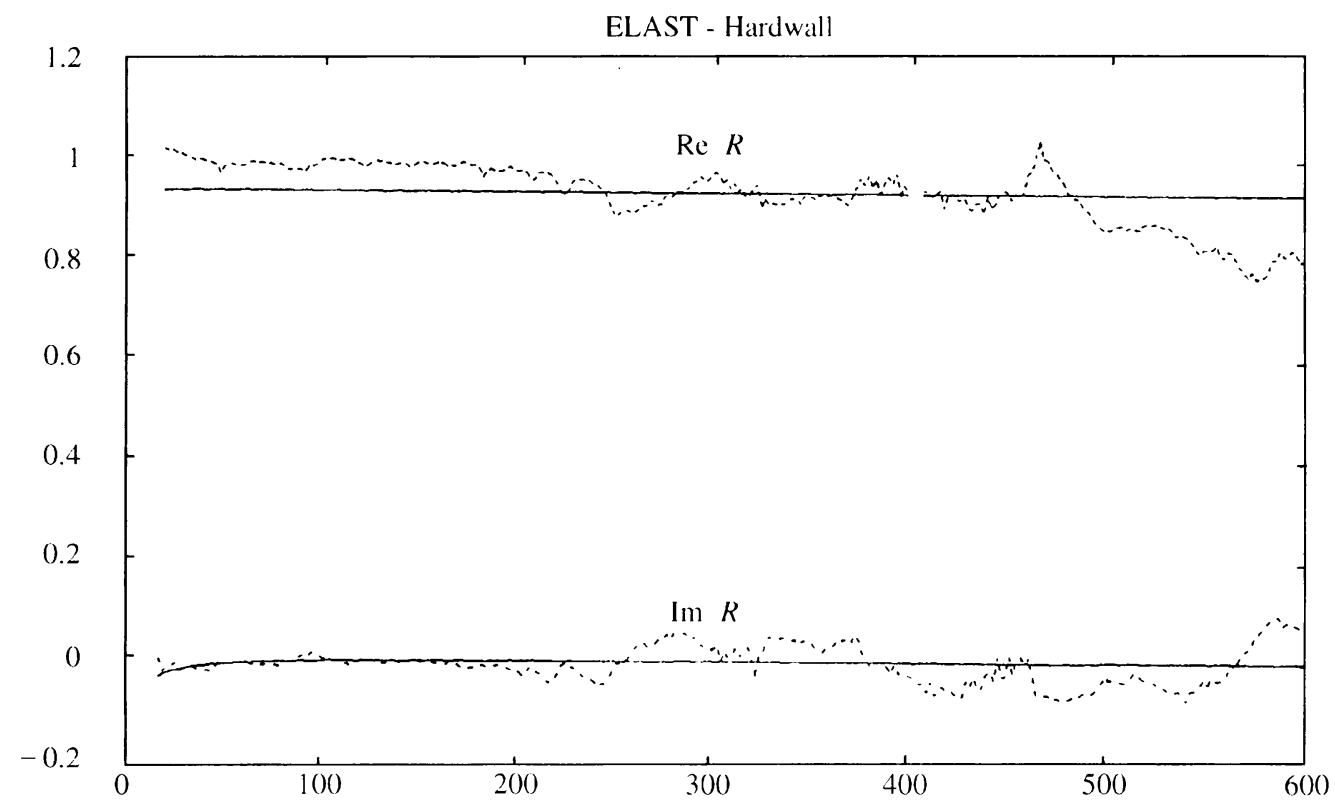

FI(i, 2l)

close to 1 at low frequencies and that decreases as the frequency is increased. The data for this case is shown in Fig. 4 (see p. 260) and may be generally regarded to exhibit the correct trends. There is, however, a noticeable dip in the real part at $77 \mathrm{hz}$. The cause for this low frequency behavior is not clear.

In addition to the above cases, the material properties of a one-inch thick acoustic foam backed by a hard surface were investigated using the impedance tube facility of reference [17]. This facility is designed for this type of measurement and better estimates of the material acoustic properties are to be expected. Data (depicted in Fig. 5 on p. 261) from these experiments is referred to as the foam termination data below, and the general trends attributed to the wedge case above may also be attributed to this case.

3.2. Computational procedures. In order to evaluate the correspondence of the mathematical models of Sec. 2 with the measured data described in Sec. 3.1, the "difference" between the measurements for varying frequency $f=\omega /(2 \pi)$ and the models' reflection coefficients $R(\omega)$ was considered. By "difference" any kind of numerical variation between the measurements and the values $R\left(\omega_{j}\right)$ could be taken. For example, if the phase of the reflected waves is of main interest (which in some sense is the case for noise suppression based on destructive interference), then "difference" could be based on deviation of the model's phase shifts arg $R$ from the measured ones.

Here, we choose as "difference" the distance of the measurements to the models' reflection coefficients as numbers in the complex plane. This seems to be a neutral choice in terms of a general comparison. To determine the best possible fit of the functions $R(\omega)$ 


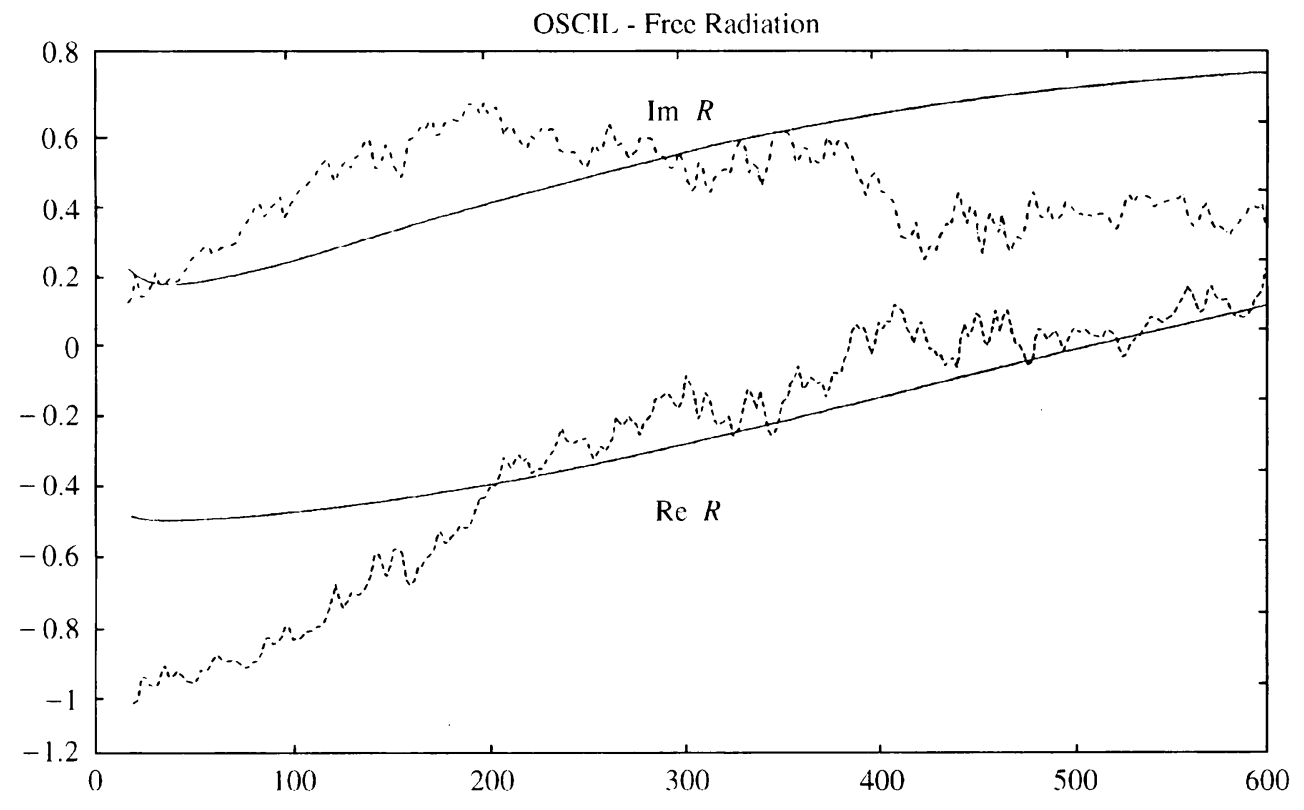

Frequency in hz

Fig. 3a

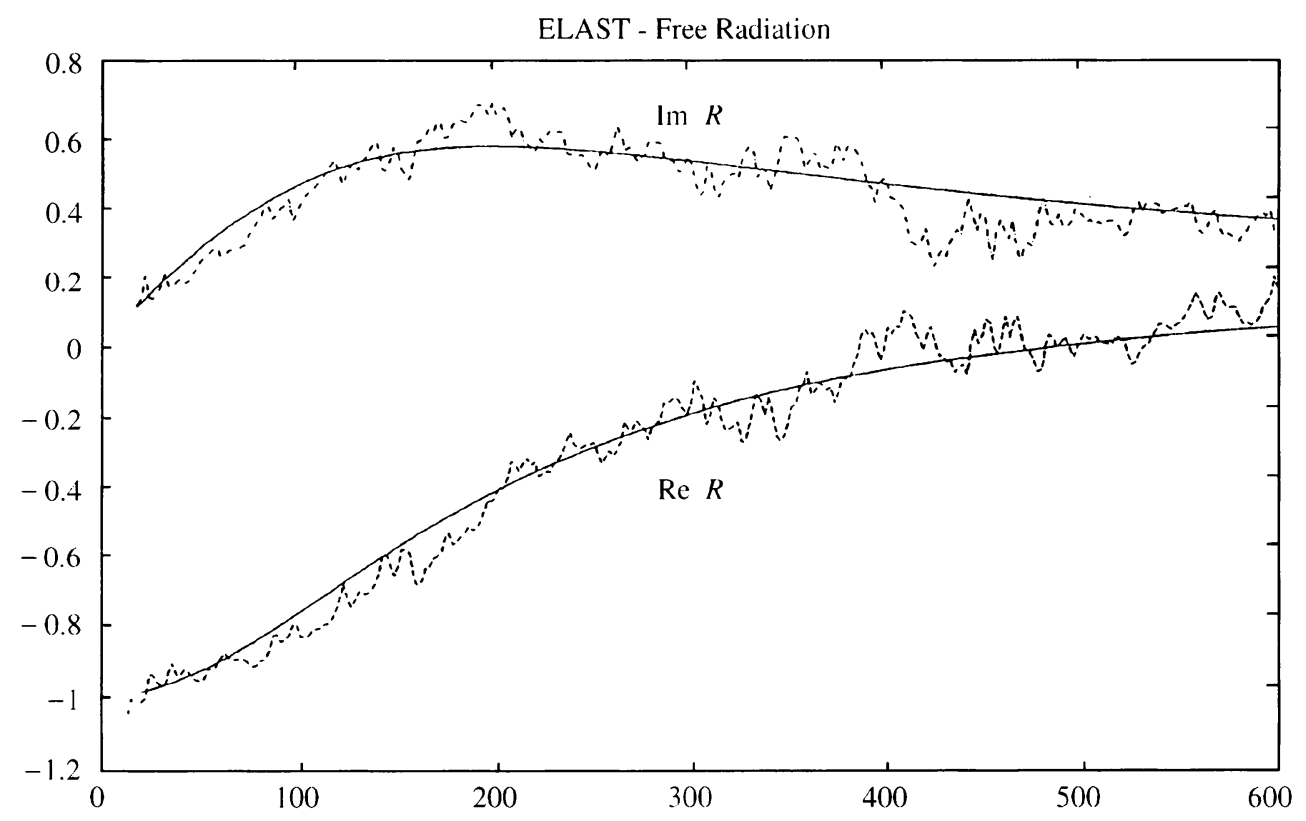

Frequency in hz

Fig, 3b 


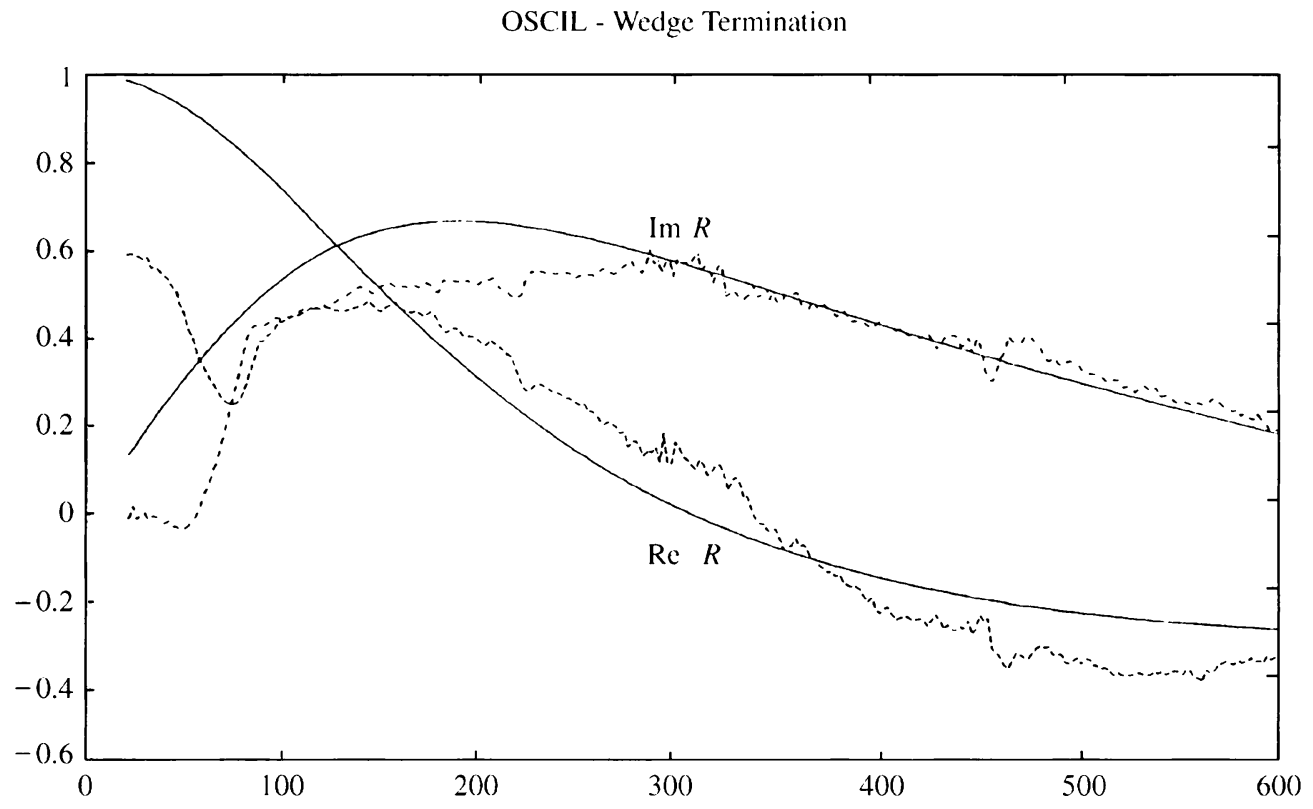

Frequency in $h z$

FIG. 4a

ELAST - Wedge Termination

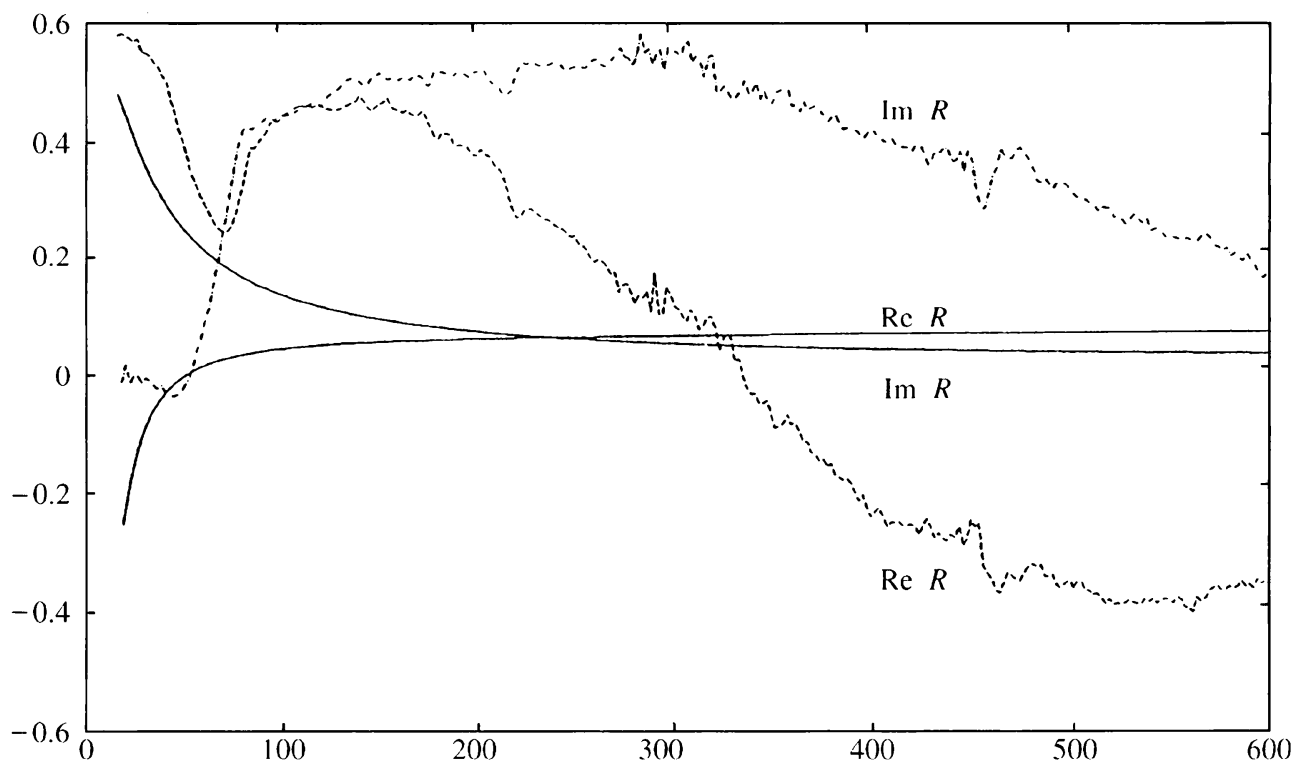

Frequency in hz

FI(i. 4 b 


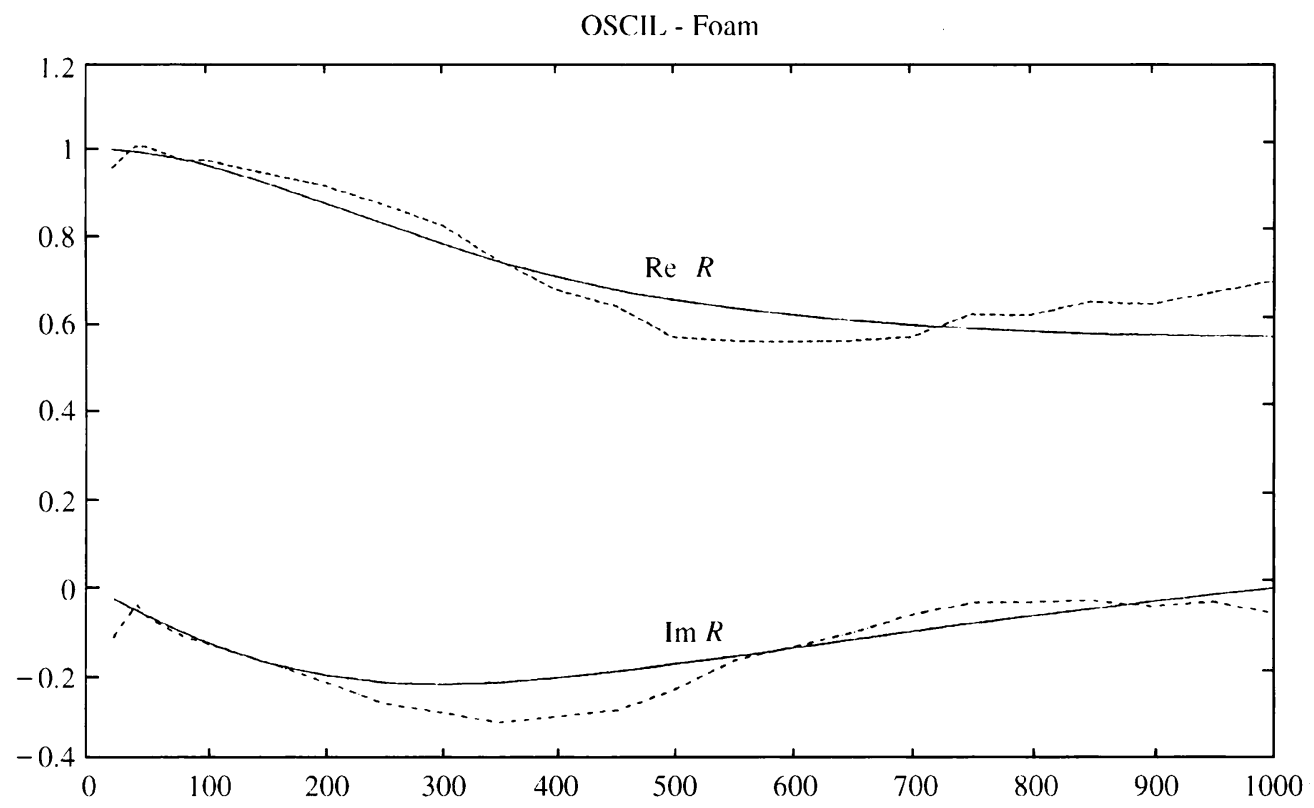

Frequency in hz

FIG. $5 a$

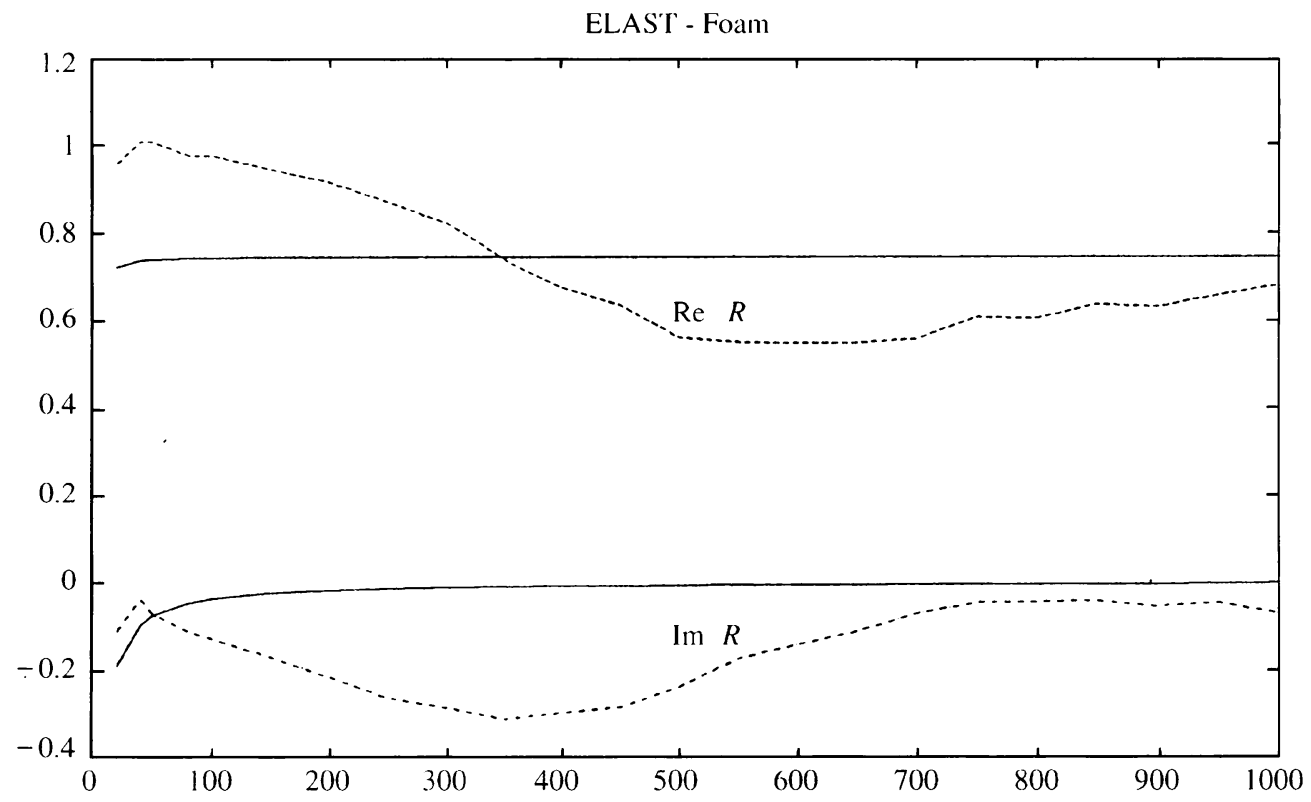

Frequency in hz.

FIG. 5b 
to the data $R_{j}$, we minimized the functional

$$
S S Q=\sum_{j=1}^{n}\left|R_{j}-R\left(\omega_{j}\right)\right|^{2}
$$

by variation of the parameters in $R(\omega)$. Here $n$ is the number of measurements $R_{j}$ at frequencies $f_{j}=\omega_{j} /(2 \pi)$ within the range of frequency considered. Because the data for the foam termination was taken in the facility of [17] at only discrete frequencies, there were only $n=23$ frequency data points available between $20 \mathrm{hz}$ and $1000 \mathrm{hz}$. For the other three experiments the range of frequency considered is $20 \mathrm{hz} \leq f_{j} \leq 600 \mathrm{hz}$ with $n=291$. For each combination of a model with a duct termination, the minimal value of $S S Q$ is an evaluation of the quality of the correspondence of the model with the data.

Note that the data that is derived from pressure measurements is also applicable to the reflection coefficients for the velocity potential, because the right and left propagating components of the pressure waves are proportional to the time derivative of the corresponding components of the velocity potential, i.e., if $\phi(t, x)=F(t-x / c)+G(t+x / c)$ is the velocity potential, then $p(t, x)=\rho \phi_{t}(t, x)=\rho\left[F^{\prime}(t-x / c)+G^{\prime}(t+x / c)\right]$, which is consistent with Eq. (3.1) if we choose $F(\xi)=A(\omega) \exp (i \omega \xi) /(i \rho \omega)$ and $G(\xi)=$ $A(\omega) R(\omega) \exp (i \omega \xi) /(i \rho \omega)$.

4. Results and discussion. The purpose of this work was to evaluate the ability of the proposed models to characterize a variety of real acoustic boundary conditions. As such there was no attempt to "smooth" the data by using analytical models or "perfect conditions" such as absolute hardwall or resonator impedance conditions in place of measured data. The data was collected for some general conditions which might be expected to be encountered in practice.

For the minimization of the sum of squares $S S Q$ over the parameters $(m, d, k) \in$ $\mathbf{R}^{3}$ and $(\alpha, \beta) \in \mathbf{R}^{2}$ we used LMDIF1 of the FORTRAN package MINPACK in the public-domain library NETLIB at Argonne National Laboratory. This routine is an implementation of the Levenberg-Marquardt algorithm with implicit scaling and optimal choice for the correction steps [18]. Starting from an initial estimate for the parameters, the algorithm converges to a local minimum. A systematic search with a variety of initial estimates and the use of graphics indicates that in all examples the numbers listed in Table 2 (see p. 264) with corresponding parameters in Table 1 are the unique global minima of $S S Q$. In one case, for (OSCIL) and the hardwall data, a second local minimum $10^{3} \times S S Q / n=4.5075$ is located at $m=-1708.4, d=9.3346, k=-15384$.

Considering (IMPED), a simple algebraic argument shows that the gradient of $S S Q$ with respect to the two real parameters $(\operatorname{Re} \zeta, \operatorname{Im} \zeta) \in \mathbf{R}^{2}$ vanishes if and only if $\operatorname{Re} R=$ $\sum \operatorname{Re} R_{j} / n$ and $\operatorname{Im} R=\sum \operatorname{Im} R_{j} / n$. Thus, to get the optimal least-squares fit for (IMPED), we compute the mean values of $\operatorname{Re} R_{j}$ and $\operatorname{Im} R_{j}$ and then $\zeta$ according to $\zeta=(1+R) /(1-R)$.

The corresponding minimal sums of squares divided by the number of data $n$ are given in Table 2.

The experimental data (measured reflection coefficients-real and imaginary parts--as a function of frequency) are represented by dashed lines in Figs. 2-5 for hard- 
TABLE 1. Minimizing parameters

\begin{tabular}{c|c|c|c|c|c|c} 
& & Hard & Frec & Wedge & Foam & Units \\
\hline \multirow{4}{*}{ OSCIL } & $m$ & 1028.0 & 114.92 & -36.493 & 85.621 & $10^{-3} \mathrm{~kg} / \mathrm{m}^{2}$ \\
& $d$ & 8.6669 & 0.1311 & 0.2189 & 1.4551 & $10^{3} \mathrm{~kg} / \mathrm{m}^{2} \mathrm{~s}$ \\
& $k$ & 13607. & -8.4717 & -788.62 & 3851.9 & $10^{3} \mathrm{~kg} / \mathrm{m}^{2} \mathrm{~s}^{2}$ \\
\hline ELAST & $\alpha$ & -2.7985 & 2178.4 & 154.17 & -16.054 & $1 / \mathrm{s}$ \\
& $\beta$ & 34.610 & 761.85 & 886.44 & 146.15 & $10^{-3}$ \\
\hline IMPED & $\operatorname{Re} \zeta$ & 28.340 & 0.3781 & 0.8073 & 4.8860 & 1 \\
& Im $\zeta$ & -3978.6 & 449.95 & 717.72 & -3349.2 & $10^{-3}$
\end{tabular}

wall, free radiation, wedge, and foam terminations, respectively. The reflection coefficients corresponding to the models with boundary conditions (OSCIL) and (ELAST) evaluated at the optimal parameters given in Table 1 are plotted in each figure by solid lines. The curves for (IMPED) are horizontal at the mean values of $\operatorname{Re} R_{j}, \operatorname{Im} R_{j}$ and are not plotted.

As might be expected, none of the above three boundary conditions can be adjusted to all four of the boundary surfaces considered. The models for the impedance or reflecting conditions are also limited in the degrees of freedom that they may exhibit. The impedance condition must by its definition be frequency independent. The elastic condition models only the prescribed frequency variation on the imaginary component. The oscillating boundary may model a somewhat more general condition as the resonant behavior may be tuned to any frequency and damped as necessary. However, one must keep in mind that there are only three real constants to adjust to model a general frequency dependence. It is clearly too much to expect that one single boundary condition will model any general acoustic boundary termination.

But there are cases where the model curves fit to the data quite well: (OSCIL) for hardwall ( $f \leq 450 \mathrm{hz}$ ) and foam termination and (ELAST) for free radiation.

The frequency dependence of the measured reflection coefficients being a structural feature of the data (except for the hardwall with $f \leq 450 \mathrm{hz}$ ), the frequency-independent boundary condition (2.10) is not an appropriate model for the terminations free, wedge, and foam, unless only a narrow range of frequency is considered.

In four examples the unconstrained minimization of SSQ renders negative parameters $(m, k, \alpha<0)$ which contradicts their physical definition and the assumptions used in the proofs of well-posedness of the models. Moreover, in these four cases the fit of the model functions to the data is not satisfying and would be worse if the positivity conditions were enforced in a minimization procedure with constraints. Thus, the oscillator boundary condition appears to be not applicable for free radiation or wedge termination nor is the damped elastic boundary condition suited for hardwall or foam termination.

The set of data from the wedge termination experiment displays a pronounced minimum at $65 \mathrm{hz}$ and then decreases nearly linearly from about $150 \mathrm{hz}$ thru $600 \mathrm{hz}$. The phase of the data set suffers the same anomaly near $65 \mathrm{hz}$ and then increases linearly from about 40 degrees to 180 degrees at $600 \mathrm{hz}$. Considering this variation, it is not 
TABLE 2. Residual sum of squares

\begin{tabular}{c|c|r|r|r}
$10^{3} \times S S Q / n$ & Hard & \multicolumn{1}{c|}{ Free } & Wedge & \multicolumn{1}{|c}{ Foam } \\
\hline OSCIL & 3.8983 & 103.735 & 51.586 & 5.116 \\
\hline ELAST & 4.8153 & 7.819 & 261.820 & 54.729 \\
\hline IMPED & 4.7972 & 134.387 & 137.038 & 36.593
\end{tabular}

surprising that the algebraically simple functions $R(\omega)$ cannot mimic this data curve when only two or three parameters can be adjusted. But this is not the main reason for the difficulties with the wedge data. When consideration of the data is restricted to the range $250 \mathrm{hz}<f<600 \mathrm{hz}$, where it is simply decreasing, the optimal mass and stiffness in (OSCIL) are again negative (the fit of Re $R$ and $\operatorname{Im} R$ increases, $10^{3} \times S S Q / n=7.049$ ) and the optimal fit of (ELAST) again is not satisfactory (alpha, beta again positive, but $10^{3} \times S S Q / n=76.009$ ). This data was modeled assuming that the reflection plane was at the duct outlet which corresponds to the back of the foam layer. From an acoustic standpoint, the face of the wedges might be considered a more physically intuitive position to take as a reference. This, however, only changes the phase response, increasing the slope significantly. Modeling the data in this way resulted in similar non-physical models and the data fit was even worse. These results suggest that the algebraic structure of the models considered here does not well represent the physical mechanisms inherent in the wedge boundary termination.

5. Conclusions. In summary, the unconstrained least-squares fits suggest that among the boundary conditions considered, several might be appropriate for use in describing the reflection of harmonic waves by the duct terminations over the range of frequency considered. For the hardwall termination. the (OSCIL) boundary condition with physically reasonable parameter values provides a good agreement of reflection coefficients in the range $f \leq 450 \mathrm{hz}$. The (OSCIL) boundary condition is also a reasonable choice for use with the foam termination. For the free radiation termination, the damped elastic conditions (ELAST) offer a good approximation of the model to the experimental data. When the reflection of waves of arbitrary shape is to be modeled, a more detailed investigation of experimental data for such waves could give additional information on the quality of these boundary conditions over a wider range of frequency.

For the wedge termination case the poor least-squares fits discussed above suggest that a more specific model for the interaction of the (non-flat) boundary surface with the interior field is needed to cover the experimentally observed phenomena.

\section{REFERENCES}

[1] P. M. Morse and K. U. Ingard. Theoretical Acoustics. NcGraw-Hill. New York. 1968

[2] IV. E. Zorunski and T. L. Parrott. Non-linear acoustic theory for rigid porous materials. NASA TN D-6196, June 1971

[3] S. I. Hariharan and H. C. Lester. A finite difference solution for the propagation of sound in near sonic flows, J. Acoust. Soc. Amer. 75. 10521061 (1984)

[4] J. S. Preisser, et al, Flight study of induced Turbofan inlet radiation with theoretical comparisons. AIAA J. of Aircraft 22. 5762 (1985) 
[5] W. R. Watson and M. K. Myers, Numerical computation of steady state acoustic disturbances in flow, AIAA paper no. AIAA-92-02-074 presented at DGLR/AIAA 14th Aeroacoustics Conference, Aachen, FRG, May $11 \cdot 14,1992$

[6] K. R. Meadows and J. C. Hardin, Removal of spurious reflections from computational fluid dynamic solutions with the complex Cepstrum, AIAA Journal 30, 29-34 (1992)

[7] B. Bolton and E. Gold, The determination of acoustic reflection coefficients by using spectral techniques, I: Experimental procedures and measurements of polyurethane foam, J. Sound Vibration 110, no. (2), 179-202 (1986)

[8] J. T. Beale, Spectral properties of an acoustic boundary condition, Indiana Univ. Math. J. 25, $895 \quad 917$ (1976)

[9] J. T. Beale and S. I. Rosencrans, Acoustic boundary conditions, Bull. Amer. Math. Soc. (N.S.) 80, 1276-1278 (1974)

[10] A. Majda, Disappearing solutions for the dissipative wave equation, Indiana Univ. Math. J. 24, $1119-1133$ (1975)

[11] G. Chen, A note on the boundary stabilization of the wave equation, SIAM J. Control Optimization 19, 106-113 (1981)

[12] H. T. Banks, S. L. Keeling, and R. J. Silcox, Optimal control techniques for active noise suppression, Proc. 27th Conf. on Decision and Control, Austin, TX, 1988, pp. 2006--2011

[13] A. Majda, The location of the spectrum for the dissipative acoustic operator, Indiana Univ. Math. J. 25, 973-987 (1976)

[14] J. Lagnese, Decay of solutions of wave equations in a bounded region with boundary dissipation, J. Differential Equations 50, 163-182 (1983)

[15] D. L. Russell, Control theory of hyperbolic equations related to certain questions in harmonic analysis and spectral theory, J. Math. Anal. Appl. 40, 336-368 (1972)

[16] H. T. Banks, G. Propst, and R. J. Silcox, Groups generated by wave-duct acoustics with impedance boundary conditions, CAMS Tech. Report \#90-10, University of Southern California, August, 1990

[17] M. G. Jones and T. L. Parrott, Evaluation of a multi-point method for determining acoustic impedance, Mechanical Systems and Signal Processing 3, no. (1), 15-35 (1989)

[18] B. S. Garbow, K. E. Hillstrom, and J. J. More, Documentation for MINPACK subroutine LMDIF1, Argonne National Laboratory, 1980 